\title{
Nonlinear optical response of a single self-assembled InGaAs quantum dot: A femtojoule pump-probe experiment
}

Cite as: Appl. Phys. Lett. 88, 203110 (2006); https://doi.org/10.1063/1.2205722

Submitted: 16 January 2006. Accepted: 20 April 2006 . Published Online: 16 May 2006

M. Wesseli, C. Ruppert, S. Trumm, H. J. Krenner, J. J. Finley, and M. Betz

ARTICLES YOU MAY BE INTERESTED IN

Spin-preserving ultrafast carrier capture and relaxation in InGaAs quantum dots Applied Physics Letters 87, 153113 (2005); https://doi.org/10.1063/1.2103399

Invited Review Article: Pump-probe microscopy

Review of Scientific Instruments 87, 031101 (2016); https://doi.org/10.1063/1.4943211

Selection rules for probing biexcitons and electron spin transitions in isotropic quantum dot ensembles

The Journal of Chemical Physics 121, 10104 (2004); https://doi.org/10.1063/1.1808414
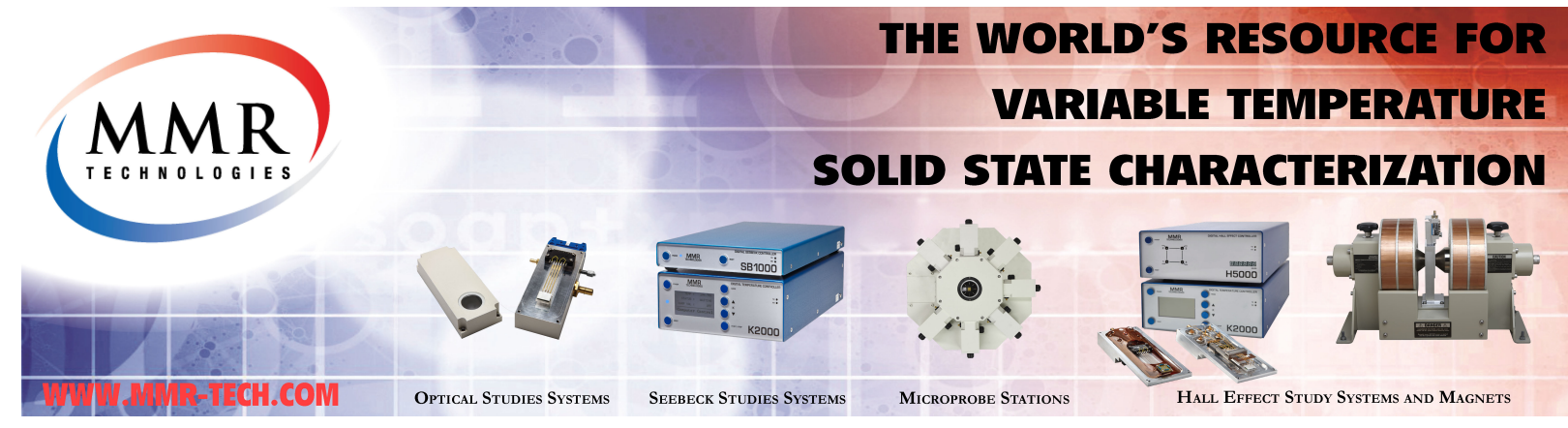


\title{
Nonlinear optical response of a single self-assembled InGaAs quantum dot: A femtojoule pump-probe experiment
}

\author{
M. Wesseli, C. Ruppert, and S. Trumm \\ Physik-Department E11, TU München, D-85748 Garching, Germany \\ H. J. Krenner and J. J. Finley \\ Walter-Schottky-Institut und Physik-Department E24, TU München, D-85748 Garching, Germany \\ M. Betz ${ }^{\mathrm{a})}$ \\ Physik-Department E11, TU München, D-85748 Garching, Germany
}

(Received 16 January 2006; accepted 20 April 2006; published online 16 May 2006)

\begin{abstract}
A single InGaAs/GaAs quantum dot is addressed in a two-color femtosecond transmission experiment in the optical near-field of a nanometer-scale shadow mask. After resonant excitation of the wetting layer beneath the nanoisland, we detect transmission changes of the quantum dot with narrow band femtojoule probe pulses. We find bleaching signals in the order of $10^{-5}$ that arise from individual interband transitions. Moreover, the nonlinear optical response reveals a picosecond dynamics associated with carrier relaxation in the quantum dot. As a result, we have developed an ultrafast optical tool for both manipulation and readout of a single self-assembled quantum dot. (C) 2006 American Institute of Physics. [DOI: 10.1063/1.2205722]
\end{abstract}

Semiconductor quantum dots (QDs) are attractive for studying the physics of quasi-zero-dimensional solid-state systems as well as for optoelectronic applications such as ultralow threshold laser devices. Especially, self-organized III-V semiconductor QDs combine remarkably long coherence times of excitonic transitions with the potential for ultrafast optical switching. ${ }^{1}$ As a result, coherently manipulating zero-dimensional nanostructures with femtosecond laser pulses is a promising concept for solid-state-based quantum information processing. ${ }^{2}$ However, most of these concepts require both ultrafast optical switching and all-optical readout of a single QD (Ref. 3) — a demanding experimental task. Until now, an analysis of the nonlinear optical response of a single QD has only been realized for the case of excitons in disordered quantum wells that typically exhibit enormous transition dipoles but only very weak confinement energies. $^{4,5}$ For isolated self-assembled QDs, Rabi oscillations have been studied extensively, thus proving the possibility of precise single qubit rotations. ${ }^{6-8}$ Moreover, decoherence times approaching the nanosecond time scale have been extracted from four-wave-mixing experiments. ${ }^{1}$ However, the time-resolved all-optical readout of a single selforganized QD remains to be demonstrated.

In this letter, we present the experimental realization of a two-color femtosecond transmission experiment of a single InGaAs/GaAs QD. The nanostructure is isolated combining a low-density growth technique with nanometer-scale aluminum shadow masks. The transmission changes of one aperture with $450 \mathrm{~nm}$ diameter are analyzed after femtosecond photogeneration of electron-hole pairs in the wetting layer beneath the QD. Most strikingly, the nonlinear optical response clearly resembles several distinct excitonic transitions of the QD under investigation. The QD typically generates modifications of the probe photon flux in the order of $10^{-5}$, thus being accessible in a shot noise-limited experimental setup. The pulse energies necessary for both the manipula-

\footnotetext{
${ }^{a)}$ Electronic mail: mbetz@ph.tum.de
}

tion and readout of the single QD are as low as a few femtojoules. The transmission changes exhibit a pronounced picosecond dynamics as recently also demonstrated for the case of an ensemble of similar QDs. ${ }^{9}$

The nanostructures of our study are InGaAs/GaAs QDs fabricated in the well-established Stranski-Krastanov growth mode. The excitonic ground state transitions of the InGaAs islands are centered around $1.35 \mathrm{eV}$. QD growth without rotation of the GaAs wafer allows to identify areas with a low QD density of a few artificial atoms per $\mu \mathrm{m}^{2}$. In addition, aluminum shadow masks are deposited on the specimen with diameters between 200 and $450 \mathrm{~nm}$. As a consequence, single QD may be precisely addressed using standard freespace optics.

In this study, we focus on a single InGaAs QD characterized in Fig. 1(b). The specimen is mounted on the cold finger of a microscope cryostat $\left(T_{L}=4 \mathrm{~K}\right)$ for all present experiments. The photoluminescence spectrum observed for a

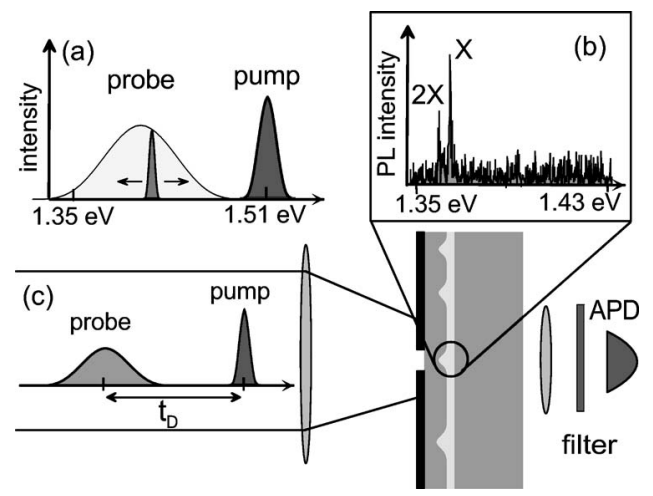

FIG. 1. (a) Excitation and probe pulses used in the experiment. The probe pulses of an energetic width of $1.2 \mathrm{meV}$ are extracted from a broadband probe continuum using a pulse shaper. (b) Photoluminescence of the nanoaperture for the case of a pulsed, low intensity excitation. (c) Scheme of the experiment: pump and probe pulse trains are focused through the metallic shadow mask of $450 \mathrm{~nm}$ diameter. The transmitted light is detected with an avalanche photodiode (APD) after spectral filtering. The sample is held at $T_{L}=4 \mathrm{~K}$ in a microscope cryostat. 
pulsed low intensity excitation at $E=1.51 \mathrm{eV}$ shows two pronounced, resolution-limited peaks. Varying the excitation power, these transitions are identified as excitonic $(X, E$ $=1.3735 \mathrm{eV})$ and biexcitonic transition $(2 X, E=1.3696 \mathrm{eV})$. The confinement energy in this QD is approximately 35 and $15 \mathrm{meV}$ for electrons and holes, respectively, i.e., much larger than for localized excitons in disordered quantum wells.

The experimental scheme is sketched in Figs. 1(a) and 1(c). Two synchronized femtosecond pulse trains with $76 \mathrm{MHz}$ repetition rate are derived from a two-color Ti:sapphire laser. ${ }^{10}$ The $100 \mathrm{fs}$ pump pulse is tuned to the absorption continuum of the wetting layer beneath the InGaAs QD, thus efficiently filling the nanostructure with nonequilibrium carriers. The 30 fs probe pulse encompasses the entire spectral range from 1.36 to $1.44 \mathrm{eV}$, giving access to both QD interband transitions and the wetting layer band edge. Before transmission through the sample, probe pulses with a spectral width of $1.2 \mathrm{meV}$ are extracted from this broadband pulse using a low-dispersive pulse-shaping technique. ${ }^{11}$ As a result, transform limited pulses of 1.5 ps duration are used as test pulses for the ultrafast transmission experiment. As depicted in Fig. 1(c), both excitation and time-delayed probe beams are focused onto the sample using a dispersion-free reflecting mirror objective with a numerical aperture of 0.4 that allows for a typical spot diameter of $1.5 \mu \mathrm{m}$. The light transmitted through the nanoaperture is collected with a microscope objective, filtered in order to reject transmitted pump photons, and analyzed with an avalanche photodiode. Subsequently, the pump induced transmission changes are extracted using a lock-in technique with a $30 \mathrm{kHz}$ modulation of the excitation pulse. The present scheme offers several distinct advantages over conventional pump-probe experiments that spectrally disperse the test pulse after transmission through the sample: (i) The probe photon flux per energy interval is kept high giving rise to an improved shot noise-limited sensitivity for the detection of ultrafast transmission changes. Simultaneously, the collection efficiency of probe light is very high. (ii) The total intensity of the test pulse can be restricted to a few femtojoules, thus efficiently suppressing probe induced optical nonlinearities. (iii) The time resolution of $1.5 \mathrm{ps}$ is sufficient to study typical relaxation phenomena in QDs.

We now turn the analysis of the nonlinear optical response of this specific quantum dot characterized in Fig. 1(b). Figure 2(b) displays the relative transmission changes observed at a time delay of $t_{D}=20 \mathrm{ps}$ after photoexcitation for two different intensities of the pump pulse. For comparison, the luminescence of the nanoaperture for these two excitation densities is depicted in Fig. 2(a). Most strikingly, we clearly identify a bleaching signal centered at the excitonic transition $X$ even for a pump intensity as low as $2 \mathrm{fJ}$. Raising the pump power to $10 \mathrm{fJ}$, the amplitude of the ground state bleaching remains at a level of $3 \times 10^{-5}$, indicating a saturation of this interband transition. As a consequence, this value may be seen as an estimate for both the maximum transmission changes associated with a single InGaAs QD under a shadow mask and the total ground state absorption in agreement with recent linear studies. ${ }^{12}$ Additionally, a positive background signal appears especially for the higher excitation level of the study which might reflect a substrate induced optical nonlinearity. Surprisingly, we do not see a clear signature of the biexciton $2 X$ in the transmission changes. For photon energies larger as compared to these

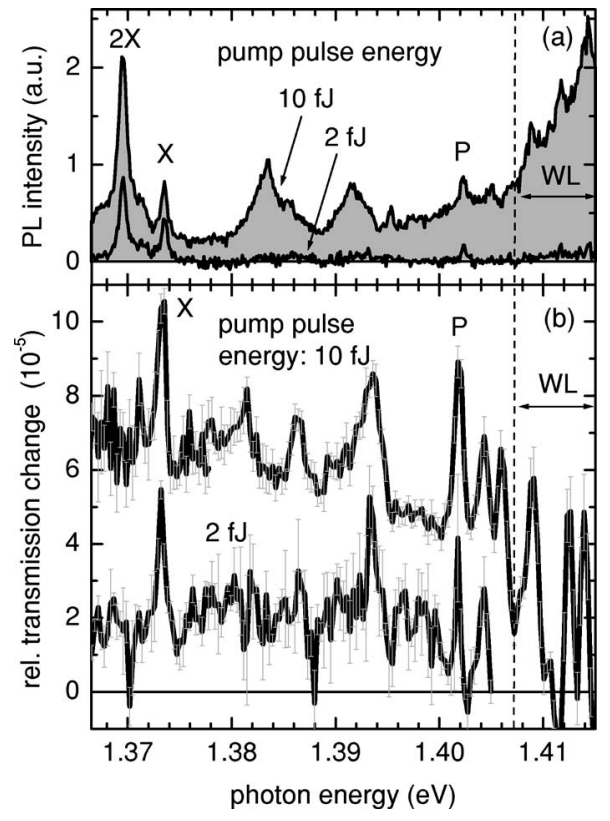

FIG. 2. (a) Photoluminescence (PL) spectrum for two different excitation intensities. The energy values indicated in the graph correspond to the pulse energy that is incident on the geometrical area of the shadow mask. (b) Transmission changes of the aperture detected at $t_{D}=20 \mathrm{ps}$ after excitation of the sample with a $100 \mathrm{fs}$ pulse centered at $1.51 \mathrm{eV}$. The spectral resolution of the experiment is determined by the energetic width of the tunable probe pulses of $1.2 \mathrm{meV}$. The data for the pump intensity of $10 \mathrm{fJ}$ are shifted upwards by two ordinate units for clarity. The solid lines represent the average obtained from ten individual scans with the vertical bars indicating the error range.

$s$-shell transitions of the QD, the luminescence spectra in Fig. 2(a) show a series of broad emission peaks that are related to optical recombination from different multiexciton complexes. These excitonic transitions partially overlap with the emission of different localized states in the twodimensional wetting layer with a band gap energy of $1.41 \mathrm{eV}$. Interestingly, most of these luminescence peaks have their direct counterpart in the nonlinear optical response. As an example, the luminescence peak $P$ in Fig. 2(a) $\left(E_{P}=1.4022 \mathrm{eV}\right)$ is paralleled by a remarkably sharp and pronounced nonlinear transmission peak in Fig. 2(b). For probe photon energies approaching the band edge of the wetting layer at $1.41 \mathrm{eV}$, we find a strongly structured nonlinear optical response that is related to different localized excitons in the wetting layer (only partially shown in Fig. 2). The amplitude of these transmission changes is one order of magnitude larger when compared to the ground state transition $X$. This finding is related to the giant transition dipole of these weakly confined excitonic complexes and is consistent with previous experiments. ${ }^{4}$

We now turn to the delay time dependence of the transmission changes discussed above. To this end, we focus on a probe photon energy interval around the transition $P$ centered at $E_{P}=1.4022 \mathrm{eV}$ (see also Fig. 2). In contrast to the ground state transition $X$, the signal-to-noise ratio in this spectral window is sufficient to study even weak differences of the transmission changes as a function of time elapsed after carrier injection. As recently verified by ensemble measurements, we expect a filling of the excited QD states within a few picoseconds and a subsequent relaxation towards the excitonic ground state on a comparable time scale. ${ }^{9}$ Figure 3 shows the transmission changes for three different delay 


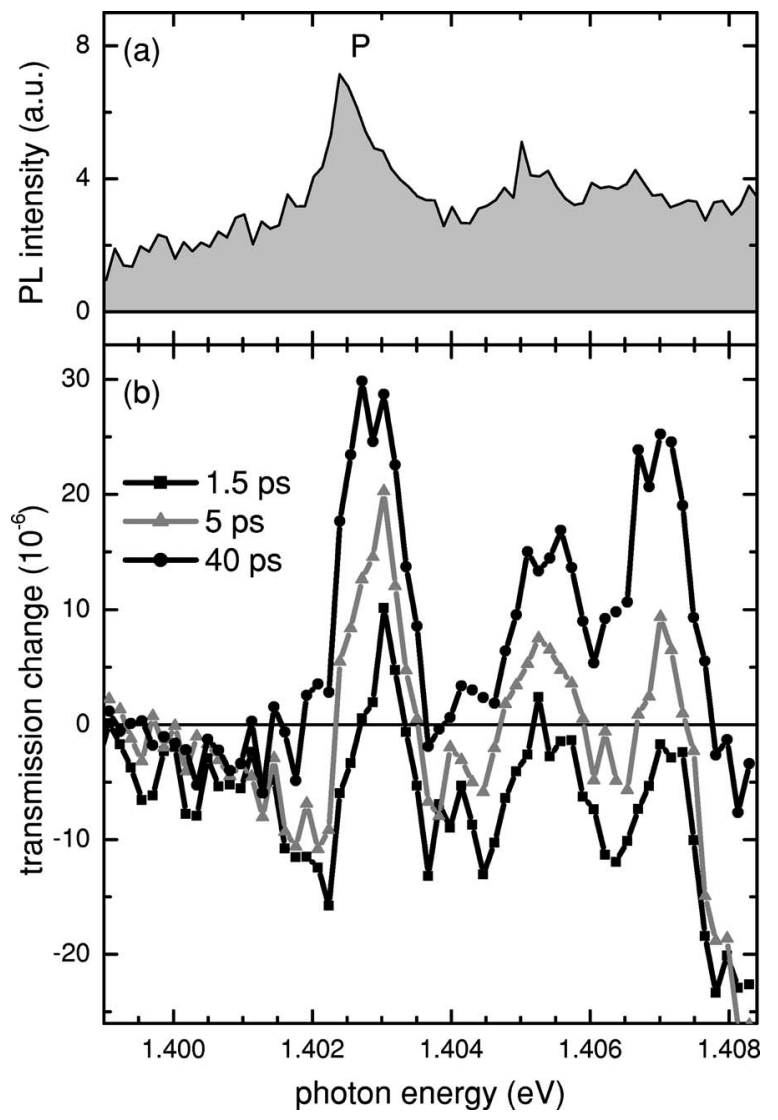

FIG. 3. (a) Photoluminescence (PL) spectrum around the peak $P$ for an excitation pulse intensity of $10 \mathrm{fJ}$. (b) Transmission changes in the corresponding spectral window detected for different delay times after photoexcitation with a $100 \mathrm{fs}$ pulse centered at $1.51 \mathrm{eV}$.

times indicated in the graph. At $t_{D}=1.5 \mathrm{ps}$, i.e., after the pump-probe overlap in the sample, we observe three bleaching peaks associated with different multiexciton configurations. Interestingly, the shape of the transmission change of the peak $P$ differs from the observed photoluminescence (PL) spectrum. As a function of the time delay after carrier injection, both the relative and the absolute intensities of the three bleaching peaks vary. Moreover, also the spectral shape of the transmission change associated with the multiexciton configuration $P$ depends on the delay time. A deeper physical picture of these results remains to be developed. We would like to note that we have not performed a systematic study as a function of temperature. However, we do not expect clear signatures of individual excitonic transitions for elevated temperatures since the confinement energy of the QDs is relatively weak. As a result, even the photoluminescence peaks suffer significant broadening with increasing lattice temperature.

In conclusion, we have demonstrated an experimental tool to both manipulate and readout a single self-assembled quantum dot on ultrashort time scales. One artificial atom beneath a shadow mask of $450 \mathrm{~nm}$ diameter is found to generate modifications of a probe photon flux in the order of $10^{-5}$, thus being accessible in a shot noise-limited detection scheme. The transmission changes clearly reflect the optical bleaching induced by different multiexciton configurations. Extending the study to resonant QD pumping, we expect deeper insight into the physics of self-organized QDs and their potential applications in quantum information processing.

The authors would like to thank A. Laubereau for valuable discussions and continuous support. This work is supported by the DFG in the framework of the Sonderforschungsbereich SFB631.

${ }^{1}$ P. Borri, W. Langbein, S. Schneider, U. Woggon, R. L. Sellin, D. Ouyang, and D. Bimberg, Phys. Rev. Lett. 87, 157401 (2001).

${ }^{2}$ N. H. Bonadeo, G. Chen, D. Gammon, D. S. Katzer, D. Park, and D. G. Steel, Science 282, 1473 (1998).

${ }^{3}$ F. Troiani, U. Hohenester, and E. Molinari, Phys. Rev. B 62, R2263 (2000).

${ }^{4}$ T. Guenther, C. Lienau, T. Elsaesser, M. Glanemann, V. M. Axt, T. Kuhn, S. Eshlaghi, and A. D. Wieck, Phys. Rev. Lett. 89, 057401 (2002).

${ }^{5}$ W. Langbein and B. Patton, Phys. Rev. Lett. 95, 017403 (2005).

${ }^{6}$ T. H. Stievater, X. Li, D. G. Steel, D. Gammon, D. S. Katzer, D. Park, C. Piermarochi, and L. J. Sham, Phys. Rev. Lett. 87, 133603 (2001).

${ }^{7}$ K. Kamada, H. Gotoh, J. Temmyo, T. Takagahara, and H. Ando, Phys. Rev. Lett. 87, 246401 (2001).

${ }^{8}$ A. Zrenner, E. Beham, S. Stufler, F. Findeis, M. Bichler, and G. Abstreiter, Nature (London) 418, 612 (2002).

${ }^{9}$ S. Trumm, M. Wesseli, H. J. Krenner, D. Schuh, M. Bichler, J. J. Finley, and M. Betz, Appl. Phys. Lett. 87, 153113 (2005).

${ }^{10}$ A. Leitenstorfer, C. Fürst, and A. Laubereau, Opt. Lett. 20, 916 (1995).

${ }^{11} \mathrm{R}$. Grote and H. Fouckhardt, Opt. Express 4, 328 (1999).

${ }^{12}$ B. Aln, F. Bickel, K. Karrai, R. J. Warburton, and P. M. Petroff, Appl. Phys. Lett. 83, 2235 (2003). 